\title{
Dispersion-managed soliton in a strong dispersion map limit *
}

\author{
P. M. Lushnikov ${ }^{1,2}$ \\ 1 Theoretical Division, Los Alamos National Laboratory, MS-B284, Los Alamos, New Mexico, \\ 87545 \\ ${ }^{2}$ Landau Institute for Theoretical Physics, Kosygin St. 2, Moscow, 117334, Russia
}

\begin{abstract}
A dispersion-managed optical system with step-wise periodical variation of dispersion is studied in a strong dispersion map limit in the framework of path-averaged Gabitov-Turitsyn equation. The soliton solution is obtained by iterating the pathaveraged equation analytically and numerically. An efficient numerical algorithm for obtaining of DM soliton shape is developed. The envelope of soliton oscillating tails is found to decay exponentially in time while the oscillations are described by a quadratic law.
\end{abstract}

OCIS codes: 060.2330, 060.5530, 060.4370, 190.5530, 260.2030.

This information is Copyright 1999 Personal TeX, Inc. All Rights Reserved.

A dispersion-managed $(\mathrm{DM})$ optical fiber is designed to create a low (or even zero) path-averaged dispersion by periodically alternating dispersion sign along an optical line which dramatically reduces pulse broadening. Recently dispersion management has become an essential technology for development of ultrafast high-bit-rate optical communication

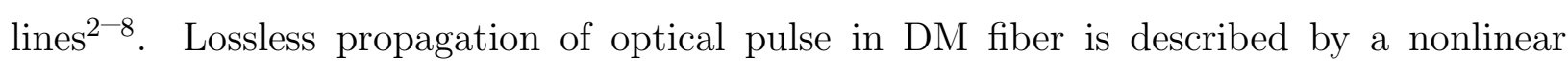

\section{*Submitted to Optics Letters}


Schrödinger equation (NLS) with periodically varying dispersion $d(z)$ :

$i u_{z}+d(z) u_{t t}+|u|^{2} u=0$

where $u$ is the envelope of optical pulse, $z$ is the propagation distance and all quantities are made dimensionless. Consider a two-step periodic dispersion map: $d(z)=\langle d\rangle+\tilde{d}(z)$, where $\tilde{d}(z)=d_{1}$ for $0<z+n L<L_{1}$ and $\tilde{d}(z)=d_{2}$ for $L_{1}<z+n L<L, L \equiv L_{1}+L_{2}$ is a dispersion map period, $\langle d\rangle$ is the path-averaged dispersion, $d_{1}, d_{2}$ are the amplitudes of dispersion variation subjected to condition $d_{1} L_{1}+d_{2} L_{2} \equiv 0$ and $n$ is an arbitrary integer number.

A nonlinearity can be treated as a small perturbation on a scales of dispersion map period $L$ provided a characteristic nonlinear length $Z_{n l}$ of the pulse is large: $Z_{n l} \gg L$, where $Z_{n l}=1 /|p|^{2}$ and $p$ is a typical pulse amplitude. Then the eq. (11) is reduced to a path-averaged Gabitov-Turitsyn 4 model:

$i \hat{\psi}_{z}(\omega)-\omega^{2}\langle d\rangle \hat{\psi}+R(\hat{\psi}, \omega)=0$

where

$$
\begin{array}{r}
R(\hat{\psi}, \omega)=\frac{1}{(2 \pi)^{2}} \int \frac{\sin \frac{s \triangle}{2}}{\frac{s \triangle}{2}} \hat{\psi}\left(\omega_{1}\right) \hat{\psi}\left(\omega_{2}\right) \\
\times \hat{\psi}^{*}\left(\omega_{3}\right) \delta\left(\omega_{1}+\omega_{2}-\omega_{3}-\omega\right) d \omega_{1} d \omega_{2} d \omega_{3},
\end{array}
$$

$\triangle \equiv \omega_{1}^{2}+\omega_{2}^{2}-\omega_{3}^{2}-\omega^{2}, s=d_{1} L_{1}$ is a dispersion map strength, $\hat{\psi} \equiv \hat{u} e^{i \omega^{2} \int_{L_{1} / 2}^{z} \tilde{d}\left(z^{\prime}\right) d z^{\prime}}$ is a slow function of $z$ on a scale $L$ and $\hat{\psi}(\omega)=\int_{-\infty}^{\infty} \psi(t) e^{\imath \omega t} d t$ is a Fourier component of $\psi$. The Gabitov-Turitsyn model is well supported by numerical simulations 8 .

Consider DM soliton solution $\psi=A(t) e^{i \lambda z}$ ( $A$ is real) of the Gabitov-Turitsyn Eq. (2) then returning to t-space one gets:

$$
\begin{array}{r}
-\lambda A+\langle d\rangle A_{t t}=\frac{1}{2 \pi s} \int C i\left(\frac{t_{1} t_{2}}{s}\right) A\left(t_{1}+t\right) \times \\
A\left(t_{2}+t\right) A\left(t_{1}+t_{2}+t\right) d t_{1} d t_{2},
\end{array}
$$

where $C i(x)=\int_{\infty}^{x} \cos x / x d x$. It was found numerically 3 that the Gaussian ansatz 
$A_{\text {Gauss }}=p \exp \left(-\frac{\beta}{2} t^{2}\right)$

where $p, \beta$ are real constants, is a rather good approximation for the DM soliton solution. Thus the Eq. (5) can be effectively used as zero approximation for solving Eq. (田) by iterations which was done in Ref. 10 for $\langle d\rangle=0$. Following 10 one can easily make a generalization for the case of small but nonzero average dispersion $\left|d_{0}\right| \ll\left|d_{1}\right|$ and obtain a set of two transcendental Eqs. from the series expansion of a first iteration in powers of $t^{2}$ :

$$
\begin{array}{r}
\lambda=-\beta\langle d\rangle+\frac{p^{2}}{2 \sqrt{3} \tilde{s}}\left(\operatorname{arcsinh} \frac{3 \tilde{s}-\imath}{2}+\text { c.c. }\right), \\
\lambda=-3 \beta\langle d\rangle \\
+\frac{2 p^{2}}{3 \tilde{s}}\left(\sqrt{\frac{\tilde{s}+\imath}{3 \tilde{s}-\imath}}+\frac{\sqrt{3}}{12} \operatorname{arcsinh} \frac{3 \tilde{s}-\imath}{2}+\text { c.c. }\right),
\end{array}
$$

where c.c. means complex conjugation. The Eqs. (6) determine the parameters $\beta, p$ of the Gaussian ansatz (5) as a functions of system parameters $\lambda, s$.

The solution of Eq. (4) was also obtained by means of iterating this Eq. numerically. $n+1$ th iteration $A^{(n+1)}$ is given by:

$\hat{A}^{(n+1)}(\omega)=Q_{n}^{3 / 2} \frac{R\left(\hat{A}^{(n)}, \omega\right)+(|\langle d\rangle|-\langle d\rangle) \omega^{2} \hat{A}^{(n)}(\omega)}{\lambda+|\langle d\rangle| \omega^{2}}$,

where the functional $R(\hat{A}, \omega)$ is defined in (3),$Q_{n}$ is a stabilizing factor given by

$Q_{n}=\left.\frac{\hat{F}^{-1}\left(\frac{\lambda+\langle d\rangle \omega^{2}}{\lambda+|\langle d\rangle| \omega^{2}} \hat{A}^{(n)}(\omega)\right)}{\hat{F}^{-1}\left(\frac{R(\hat{A}, \omega)}{\lambda+|\langle d\rangle| \omega^{2}}\right)}\right|_{t=0}$

and $\hat{F}^{-1}$ is a Backward Fourier transform. This numerical iteration scheme was also used in Ref. 1 except that a Petviashvili stabilizing factor ${ }^{12}$ was used there instead of $Q_{n}$. But both stabilizing factors results in the convergence of iteration scheme to the same solution of (伍).

The main obstacle in numerical iteration scheme (6), (7) is the computation of integral term $R(\hat{A}, \omega)$ which generally require $N^{3}$ operations for each iteration, where $N$ is a number of grid points in $\omega$ or $t$-space. Here we introduce much more efficient numerical algorithm for calculation of $R(\hat{A}, \omega)$.

Rewriting the kernel of $R(\hat{\psi}, \omega)$ via parametric integral: 
$\frac{\sin \frac{s \triangle}{2}}{\frac{s \triangle}{2}}=\frac{1}{s} \int_{-s / 2}^{s / 2} \exp \left(i s^{\prime} \triangle\right) d s^{\prime}$

and using definition of $\triangle$ one gets from (3) :

$$
\begin{array}{r}
R(\hat{A}, \omega)=\frac{1}{s(2 \pi)^{2}} \int_{-s / 2}^{s / 2} d s^{\prime} e^{-i s^{\prime} \omega^{2}} \int \hat{A}^{\left(s^{\prime}\right)}\left(\omega_{1}\right) \\
\times \hat{A}^{\left(s^{\prime}\right)}\left(\omega_{2}\right) \hat{A}^{\left(s^{\prime}\right) *}\left(\omega_{3}\right) \delta\left(\omega_{1}+\omega_{2}-\omega_{3}-\omega\right) d \omega_{1} d \omega_{2} d \omega_{3},
\end{array}
$$

where $\hat{A}^{\left(s^{\prime}\right)}(\omega) \equiv \hat{A}(\omega) e^{i s^{\prime} \omega^{2}}$. In $t$-space this expression takes the form

$\hat{F}^{-1}(R(\hat{A}, \omega))=\frac{1}{s} \int_{-s / 2}^{s / 2} d s^{\prime} \mathbf{G}^{\left(s^{\prime}\right)}\left(\Psi^{\left(s^{\prime}\right)}(t)\right)$

where $\Psi^{\left(s^{\prime}\right)}(t) \equiv\left|A^{\left(s^{\prime}\right)}(t)\right|^{2} A^{\left(s^{\prime}\right)}(t)$ and $\mathbf{G}^{\left(\mathbf{s}^{\prime}\right)}$ is an integral operator corresponding to a multiplication operator $\hat{\mathbf{G}}^{\left(s^{\prime}\right)}\left(\hat{\Psi}^{\left(s^{\prime}\right)}(\omega)\right) \equiv e^{i s^{\prime} \omega^{2}} \hat{\Psi}^{\left(s^{\prime}\right)}$ in $\omega$-space. It follows from the Eqs. (9), (10) that numerical procedure for calculation of $R(\hat{A}, \omega)$ includes four steps:

(i) The Backward Fourier Transform of $\hat{A}^{\left(s^{\prime}\right)}(\omega)=\hat{A}(\omega) e^{i s^{\prime} \omega^{2}}$ for every value of $s^{\prime}$.

(ii) A calculation of $\Psi^{\left(s^{\prime}\right)}(t)$ from $A^{\left(s^{\prime}\right)}(t)$.

(iii) The Forward Fourier Transform of $\Psi^{\left(s^{\prime}\right)}(t)$.

(iv) A numerical integration (summation) of $e^{i s^{\prime} \omega^{2}} \hat{\Psi}^{\left(s^{\prime}\right)}(\omega)$ over $s^{\prime}$ for every value of $\omega$.

The Forward and Backward Fourier Transforms were performed with the Fast Fourier Transform which requires $N \log _{2}(N)$ operations. A total number of operations for one iteration is about $4 M N \log _{2}(N)$, where $M$ is a number of grid points for integration over $s^{\prime}$. We used the following typical values for numerical solution of (4): $N=8192 ; M=$ 800. One iteration on Alpha $500 \mathrm{MHz}$ workstation requires about 30 seconds for 16 -bytes (32 digits) precision. Thus numerical scheme (i)-(iv) provides dramatic improvement of numerical performance. $8192^{3}$ operations would takes 30 days on the same workstation. Note that the proposed efficient numerical algorithm can be generalized to include optical fiber losses and amplifiers.

Fig.1a,b show the dependence of a root mean square pulse width $T_{R M S} \equiv$ $\sqrt{\int t^{2} A^{2} d t / \int A^{2} d t}$ on a quasimomentum $\lambda$ obtained from $(i)$ the first iteration of the Eq. (4) using values of $\beta, p$ resulting from the Eqs. (6) (dotted curves); (ii) a variational approach 
(see e.g. Eqs. (13), (14) in Ref11) represented by dashed lines; (iii) a full numerical solution of the Eq. (4) (solid lines). The explicit expression $T_{R M S}=1 / \sqrt{2 \beta}$ for the Gaussian pulse shape is used for calculation of dashed curves. The solid curve is shown in Fig. 1b only for upper branch I of solution because numerical iteration scheme for negative average dispersion $\langle d\rangle=-0.01$ diverges on lower branch II which is in agreement with Ref.11. We also calculated a time-averaged optical power $P \equiv \int A^{2} d t$ and found that $P(\lambda)$ dependence following from the first iteration and the variational approach Ref.11 reproduce a full numerical solution of the Eq. (4) with high accuracy $(\sim 1 \%)$. One can conclude that both the Eqs. (6) and the variational approach 11 predict $P(\lambda)$ with a high accuracy while $T_{R M S}(\lambda)$ dependence is reproduced by the first iteration of the Eq. (4) with better accuracy $(\sim 2 \%)$ compare with accuracy $(\sim 40 \%)$ of the variational approach.

There is an essential difference of our numerical simulation in comparison with numerical results of Ref.11 concerning upper branch I for the negative average dispersion. After about 50 iterations of the Eq. (4) a numerical instability was detected on the tails of DM soliton for $\langle d\rangle=-0.01$. (Presumably this instability was not found in Ref 11 because a few iterations was considered there). A finer numerical grid slows down numerical instability growth but does not kill it. The instability slows down as $\langle d\rangle \rightarrow 0$ and for $\langle d\rangle \geq 0$ there is no numerical instability. Thus the solid curve in Fig. 1b for $\langle d\rangle=-0.01$ can only be formally attributed to DM soliton and the question about existence of DM soliton for the negative average dispersion is still open. It is possible that the instability within the numerical iteration scheme results from a resonance of DM soliton tails with linear waves11. But there is another alternative that DM soliton solution does not exist for any negative average dispersion value and instead of DM soliton on can observe a long-lived quasi-stable structure. Note that the existence of DM soliton for nonnegative average dispersion for the Eq. (4) was proved in Ref.13. In addition it was proved in Ref 14 that even if DM soliton exists for $\langle d\rangle<0$ it can not realize a minimum of the Hamiltonian of the Eq. (2) for fixed $P$. This can indicate that DM soliton is unstable in that case. Related result 15 is the nonexistence criterion for a periodic solution of the Eq. (11) for a negative enough average dispersion. But Refs.13 15 do 
not give any statement about the existence of DM soliton for small negative $\langle d\rangle$.

Fig. 2 shows a typical shape of DM soliton. This is the first to the best of my knowledge high precision numerical solution of the Eq. (四). Note that solid curve dips do not reach $t$-axes only because of finite size of numerical grid. On can conjecture from Fig. 2 the asymptotic of DM soliton is given by

$A_{\text {asymp }}(t)=f(t) \cos \left(t^{2}\left[a_{0}+a(t)\right]\right) \exp (-b|t|)$

where $a_{0}, b$ are constants and $f(t) /|t|, a(t)$ are slow functions of $t$. An analysis of fast oscillations in integral term of the Eq. (4) allows to show that $f(t)=c|t|+O(1), a_{0}=1 / 2 s, a(t)=$ $a_{1} /|t|+a_{2} / t^{2}+O\left(1 /|t|^{3}\right)$ for $|t| \rightarrow \infty,\langle d\rangle \rightarrow 0$, where $c, a_{1}, a_{2}$ are constants. Dashed curve in Fig. 2 shows $A_{\text {asymp }}^{2}(t)$ dependence for $c=11.9654, b=3.04515, a_{1}=1.41364, a_{2}=1.51023$ which is in very good agreement with asymptotic of numerical solution of the Eq. (4) (solid curve). Thus the envelope of DM soliton oscillating tails decays exponentially while the oscillations are described by a quadratic law. Detailed consideration of the asymptotic solution is outside the scope of this Letter.

The author thanks M. Chertkov, I.R. Gabitov, E.A. Kuznetsov and V. Zharnitsky for helpful discussions.

The support was provided by the Department of Energy, under contract W-7405-ENG36, RFBR (grant 00-01-00929) and the program of government support for leading scientific schools (grant 00-15-96007).

E-mail address: lushnikov@cnls.lanl.gov 


\section{REFERENCES}

1. C. Lin, H. Kogelnik and L.G. Cohen, Opt. Lett., 5, 476 (1980).

2. M. Nakazawa, H. Kubota, Electron. Lett., 31, 216 (1995).

3. N.J. Smith, F.M.Knox, N.J. Doran, K.J. Blow and I. Bennion, Electron. Lett., 32, 54 (1996).

4. I. Gabitov and S.K. Turitsyn , Opt. Lett., 21, 327 (1996); JETP Lett., 63, 861 (1996).

5. S. Kumar and A. Hasegawa, Opt. Lett., 22, 372 (1997).

6. T. Lakoba and D.J. Kaup, Electron. Lett. 34, 1124 (1998).

7. P.V. Mamyshev and N.A. Mamysheva., Opt. Lett., 24, 1454 (1999); L.F. Mollenauer, P.V. Mamyshev, J. Gripp, M.J. Neubelt, N. Mamysheva, L. Grüner-Nielsen and T. Veng, Opt. Lett., 25, 704 (1999).

8. S.K. Turitsyn, T. Schäfer, N.J. Doran, K.H. Spatschek and V.K. Mezentsev, Opt. Comm. 163, 122 (1999).

9. M.J. Ablowitz and G. Biondini, Opt. Lett., 23, 1668 (1998).

10. P.M. Lushnikov, Opt. Lett. 25, 1144 (2000).

11. D.E. Pelinovsky, Phys. Rev. E 62, 4283 (2000).

12. V.I. Petviashvili and O.A. Pokhotelov, Solitary Waves in Plasmas and in the Atmosphere (Gordon and Breach, Philadelphia, 1992), p. 248.

13. V. Zharnitsky, E. Grenier, S.K. Turitsyn, C.K.R.T. Jones and J.S. Hesthaven, Phys. Rev. E 62, 7358 (2000).

14. V. Zharnitsky, E. Grenier, C.K.R.T. Jones and S.K. Turitsyn, to appear in Physica D (2001).

15. P.M. Lushnikov, JETP Lett. 72, 111 (2000). 


\section{Figure captions:}

Fig.1. $T_{R M S}$ for $s=1,\langle d\rangle=0.01$ (a) and $\langle d\rangle=-0.01$ (b). Branches I, II for $\langle d\rangle=-0.01$ correspond to two branches of analytical solution.

Fig.2. DM soliton shape (curve 1) versus Eq. (11) (curve 2) for $\langle d\rangle=0, s=1, \lambda=1 . A(t)$ is an even function. 


\section{FIGURES}

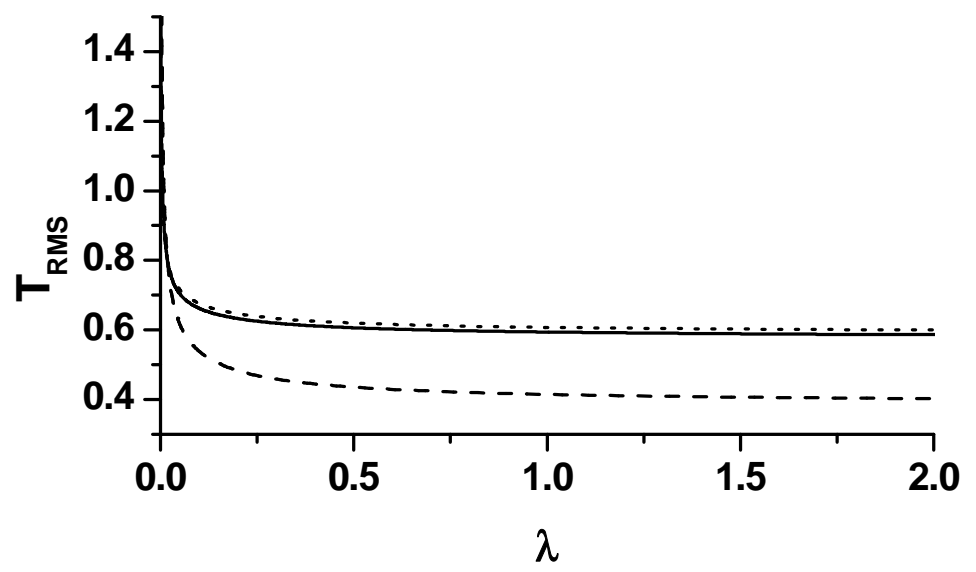

Fig. 1a

P.M. Lushnikov "Dispersion-managed soliton in a strong ..." Submitted to Opt. Lett. 


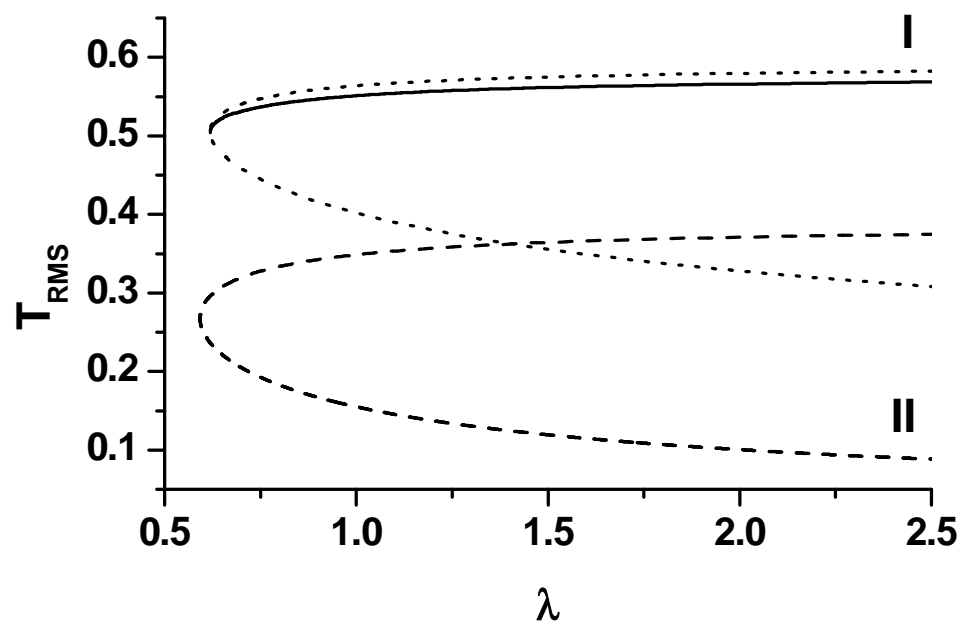

Fig. 1b

P.M. Lushnikov "Dispersion-managed soliton in a strong ..." Submitted to Opt. Lett. 


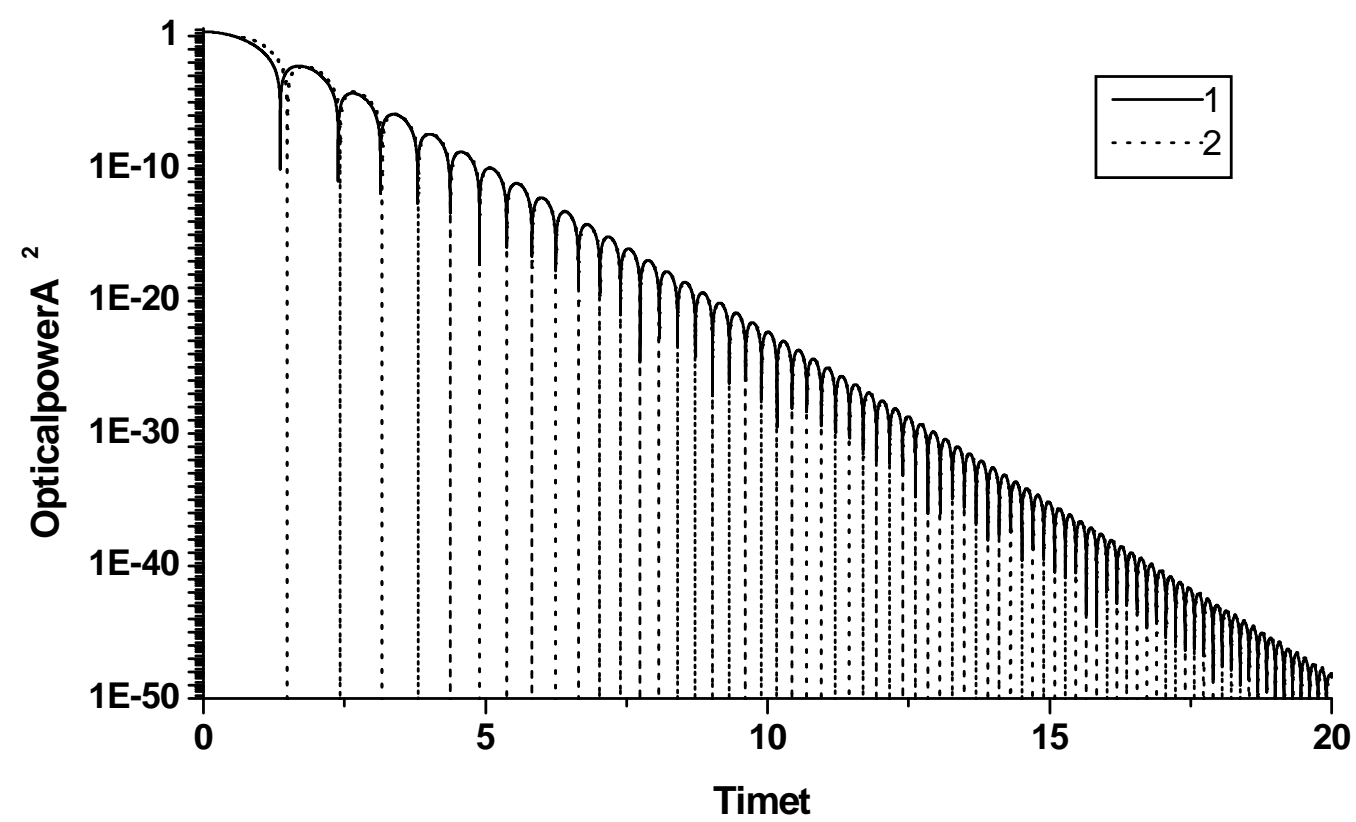

Fig. 2

P.M.Lushnikov "Dispersion-managed soliton in a strong ..." Submitted to Opt. Lett. 